\title{
Les « Blogs des indignés » ou la mise en scène de la colère de la Cité
}

\section{"Blogs of the Outraged" or a show of public anger}

\author{
Wojciech Prażuch \\ Université Pédagogique de Cracovie \\ prazuch.wojciech@gmail.com
}

\begin{abstract}
The paper aims to discuss the means of expressing and inferring emotions using as an example the lexical and iconic layers of the blogosphere of the French Outraged movement. The analysis is based on a corpus of content taken from a dozen blogs written in France between 2010 and 2013. In the course of the analysis based on theoretical assumptions of modern discourse and argumentation analysis, the author concludes that the expression of outrage in the texts under investigation is a kind of emotional form which the active co-authors of the blogs actualize in their own manner and by doing so they create not so much pathos but rather ethos - both individual and communal, based on a system of shared values and beliefs. On the other hand, argumentation through emotions usually takes place indirectly, mostly by means of axiologically charged subjectification of discourse.
\end{abstract}

Keywords: political discourse, emotion

\section{INTRODUCTION}

Les travaux de recherche récents montrent un intérêt grandissant pour une approche pluridisciplinaire des émotions. Le présent article entend s'inscrire dans cette orientation pour analyser les manifestations de l'indignation citoyenne répertoriées dans la production icono-textuelle des blogs des "Indignés » qui se réclament du fameux pamphlet de Stéphane Hessel. La réflexion engagée entend se focaliser sur la problématique de la dimension émotive de ce type de discours et plus précisément du recours aux émotions dans l'argumentation et la mobilisation collective.

Le texte est organisé en trois parties. Dans un premier temps, nous voudrions donner quelques précisions sur les émotions et le cadre méthodologique de 
l'analyse du discours émotif. Dans une seconde partie, nous passerons en revue les caractéristiques discursives des blogs militants pour montrer en quoi le support communicatif conditionne l'apparition des effets de pathémisation. Enfin, la troisième et dernière partie sera centrée sur le rôle des composantes linguistiques et para-linguistiques de la communication de l'indignation. Le premier niveau de l'analyse équivaut à la recherche qualitative par études de cas ayant pour but de dépister tout élément induisant l'état émotionnel. Nous interrogerons ensuite l'aspect pragmatique c'est-à-dire la prise en compte du contexte de parole et du type d'arguments que la formation discursive représentée peut considérer comme valables pour déclencher une émotion. On essaiera de montrer aussi l'importance des émotions en tant qu' « énergie pour l'action » pour reprendre l'expression de Cigada (2008 : 23) c'est-à-dire dans les processus de la mobilisation collective. L'hypothèse principale est que, dans le corpus étudié, le recours au terme emblématique "indignation» participe davantage du travail du pathos dans une sorte de jeu spéculaire par lequel on tente de renvoyer à l'interlocuteur une image positive de lui-même en tant que membre d'une communauté, tandis que les effets émotifs en tant que tels sont recherchés à travers d'autres procédés.

\section{INDIGNATION ET TYPOLOGIES DES ÉMOTIONS}

Qu'ils soient individuels ou collectifs, les phénomènes émotifs font tous partie de l'«éprouvé » et traduisent les modalités complexes de l'implication affective. Il y a des façons très diverses de les classer selon les disciplines ou les critères de catégorisation ${ }^{1}$. Généralement parlant, leur spectre est représenté sur l'axe de trois dimensions : intensité - durée - plaisir (euphorique/dysphorique). Mais, on peut également faire appel à des critères tels que le degré d'universalité par rapport aux différents contextes sociétaux, les composantes somatiques ou cognitives; à la distinction entre les émotions dites affectives (tristesse), informatives (ennui) ou appréciatives (colère), réactionnelles et mobilisatrices. Enfin, et c'est la question qui fait couler de l'encre depuis des siècles, on peut prendre en compte leur degré de rationalité (selon l'axe indignation/angoisse). La prétendue incompatibilité de l'émotif et du rationnel a été longtemps considérée comme une sorte de paradoxe de l'argumentation. En effet, la motivation humaine n'avait pour certains qu'une seule dimension, à savoir celle du calcul rationnel tandis que les émotions y étaient une sorte de « désordre de la

\footnotetext{
${ }^{1}$ Ainsi, dans la psychologie, on distingue entre les comportements de nature caractérielle et les réactions comportementales - jouées ou réelles. Jasper (1998) parle des émotions (réactives et temporaires) et des affects (plus durables et profonds). Christian Plantin distingue de son côté entre humeurs, sentiments, affections, passions et émotions (Plantin, 1998).
} 
conduite » (Plantin, 1998). Notons aussi que dans le domaine socio-politique, les émotions sont longtemps demeurées une sorte de tabou à cause du postulat d'irrationalité des mobilisations collectives (cf. Taine). Aujourd'hui, en revanche, de nombreux spécialistes remettent à l'honneur leur rôle de significations sociales partagées ${ }^{2}$.

$\mathrm{Au}$ regard des nombreux critères, le mot « indignation » - chez Aristote complémentaire par rapport à la pitié - peut être caractérisé comme désignant une émotion violente suscitée au contact avec la réalité (dans le cas du bonheur immérité et plus largement du sentiment d'injustice). En ce sens, elle peut être aussi une forme d'imitation affective ce qui veut dire que nous l'éprouvons par imitation des sentiments des victimes. Elle couvre tout un continuum de nuances qui vont du dégoût à la frustration, mais quelle qu'en soit l'intensité ou la nature, l'indignation est loin d'exclure la raison puisqu'elle repose sur une appréciation de la situation et qu'on connaît « objectivement» les causes du désarroi. Qui plus est, il semble légitime de croire que dans le délibératif, l'indignation ne doit pas être forcément déterminée causalement par un quelconque stimulus. Soulignons enfin son orientation actionnelle : rangée du point de vue sociologique dans la catégorie d'émotion-norme-jugement, extérieure à l'individu et contraignante, elle sert souvent de motivation pour une action revendicative.

\section{CHOIX MÉTHODOLOGIQUES}

Avant de procéder à l'analyse du matériel des blogs des Indignés, précisons le choix des cadres théoriques et méthodologiques. La démarche que nous proposons s'articule autour d'une réflexion plus générale portant sur la communication verbale et non verbale des émotions. Il n'y a pas lieu ici de brosser un panorama de théories et de postulats critiques émanant de linguistes, sémioticiens et analystes du discours (se référer à ce propos à KerbratOrecchioni, 2000) qui remontent à l'étude linguistique des marques de l'émotion chez Bally et à la fonction expressive de Jakobson. Il importe de remarquer cependant que la problématique des émotions n'est plus envisagée uniquement dans le cadre d'un processus «stimulus - réaction » et les travaux récents portent, au-delà l'approche lexicale, sur le phénomène de l'inférence et les dimensions contrastive (Wierzbicka) ou interactionniste.

Dans le discours délibératif, la position émotionnelle ne pourrait être envisagée comme un phénomène à part. Au contraire, il faut la considérer comme condition préalable à tout procédé persuasif à des fins sociopragmatiques. Pour les besoins de notre analyse, nous adoptons donc comme la

\footnotetext{
${ }^{2}$ Voir à ce propos les travaux de Jasper (1997), de Goodwin (2001) ou de Traïni (2009).
} 
plus pertinente, la lignée théorique de travaux sur la construction argumentative des émotions, et le cadre du système topique des émotions tel que proposé par Plantin (2011) et Micheli (2008 et 2010) ${ }^{3}$, mais aussi du positionnement théorique de Ruth Amossy (2006). S'inspirant des travaux de sociologues, Plantin et Micheli analysent le fonctionnement discursif des émotions en essayant de montrer qu'elles peuvent être créées ou maniées au fil du discours, et que l'organisation du matériel sémiotique peut être orientée vers la construction de telle ou telle émotion chez l'auditoire. Ce point de vue s'apparente à celui d'une rhétorique de la visée d'effet mise en place à travers les catégories de discours (inventio, dispositio, elocutio). Charaudeau considère ainsi que le point de vue discursif sur les émotions est spécifique puisqu'il ne s'intéresse pas tant à l'éprouvé, mais au «calcul» des effets émotifs mis en place dans un échange interlocutoire. D'ailleurs, si nous recourons systématiquement, à l'instar de Charaudeau, au néologisme "pathémique», c'est que dans la tradition, la notion de "pathe » renvoie aux émotions qu'un orateur emploie pour agir sur les esprits. Elle désigne donc des unités sémantiques du domaine passionnel en inscrivant en même temps l'analyse dans l'approche rhétorique du problème (Charaudeau, $2000: 137$ ).

Enfin, dans le cadre de cette étude, il ne faut pas non plus perdre de vue l'optique de la sociologie des émotions. Il s'agit notamment du rôle des émotions collectives - réciproques et partagées - présentes dans les dynamiques internes des groupes militants. Comme nous l'avons remarqué, les émotions obéissent aux normes et aux manières sociales de sentir qui sont "douées d'une puissance impérative et coercitive» en vertu de laquelle elles s'imposent à l'individu (E. Durkheim, Les règles de la méthode sociologique, cité par Morin, 2003 : 102).

\section{CONTRAINTES DU DISPOSITIF COMMUNICATIF. CARACTÉRISTIQUE GÉNÉRIQUE ET DISCURSIVE DES BLOGS MILITANTS}

Puisque toute catégorie d'effet dépend des circonstances dans lesquelles elle apparaît, le discours qui s'inscrit dans une situation de communication (Charaudeau, 2000 : 138) est déterminé en partie par le canal de transmission, les proxémies, etc., bref, un jeu de contraintes et de libertés énonciatives. C'est dans cet univers, entendu comme l'espace laissé disponible, que l'instance d'énonciation réalise une mise en scène discursive à visée pathémisante. Il semble donc légitime de présenter brièvement les principales caractéristiques que revêtent les blogs militants en tant que « support des signifiants, eux-mêmes

\footnotetext{
${ }^{3}$ Cf. aussi d'autres auteurs : K. Lund et G. Niccolai.
} 
support des significations » (Kerbrat-Orecchioni, 1999: 31). Nous nous concentrons sur trois traits ayant une incidence certaine sur la nature du discours et sa charge émotive.

Notre corpus d'analyse, homogène du point de vue de situation de communication, est composé de données extraites d'une quinzaine de blogs existant entre 2011 et 2013 et choisis en fonction de certains critères tels que l'existence locale et le trafic considérable de contributions mises en ligne ${ }^{4}$. L'analyse ne porte cependant que sur la première strate textuelle et/ou iconique, sans prendre en considération les articles de presse, les images ou autre contenu vers lequel le lecteur pourrait être renvoyé via les liens hypertexte.

Force est de constater que les blogs constituent un support qui par son caractère multiforme constitue l'extension considérable de l'acception du média. Le premier trait du canal de communication est son côté séquentiel (en formats fragmentés) et composite. Dans leur entreprise collective du mash-up de textes médiatiques, d'informations et d'images "glanées » sur le web, les internautes ont recours à des formats sémiotiques d'autant plus culturellement hybrides qu'il s'agit d'un cadre transnational et interconnecté (Broudoux, 2007). L'une des incidences de cet état de choses consiste dans le fait que les indices sociaux présents dans toute communication ont tendance à être brouillés ce qui rend difficile toute analyse des émotions dans une optique diastratique.

Deuxièmement, ces nouvelles formes internationalisées de militantisme représentent le courant contre-hégémonique du champ politique (MonnoyerSmith, 2011). Cela revient à dire que les citoyens actifs qui critiquent le monopole discursif des grands médias s'emparent des ressources de symbolisation de la société en cherchant à créer un discours parallèle. Ils réclament une « démocratie sémiotique » et attirent en même temps l'attention sur des facettes de la réalité considérées comme peu représentées voire cachées. Sans nier les vertus de la nouvelle agora électronique fournissant ses « interprétants » à la vie démocratique (cf. Céfaï et Pasquier, 2003) et de leur aspect " participatif», il faut aussi attirer l'attention sur son aspect « infopollution » dû à la faible qualité des informations (Serres, 2005).

Soulignons enfin que dans les blogs analysés, les relations discursives à priori antagoniques du fait de la radicalité délibérée des prises de position proche de celle du pamphlet - sont souvent mitigées non sans conséquence pour la charge émotive de ces écrits en ligne.

1. Mais ici, en aucun cas il ne faut être diffamatoire ou injurieux !!!

2. Témoignez, Racontez, Commentez, Exprimez vous en toute liberté ! Sachant que ses seules limites sont l'insulte, la Diffamation et le Respect de la Vie Privée.

${ }^{4}$ Le blog des indignés jurassiens, Le blog des Indignés de Guérande, Les Indignés des Combrailles, Le blog des toulonnais indignés, etc. 
Il semble par ailleurs que la parole pamphlétaire ait migré ces dernières années vers les services de réseautage social qui représentent un tout autre niveau de l'espace semi-public du web.

\section{4. ÉMOTION DÉNOTÉE / EXPLICITE}

En fonction des stratégies utilisées, l'indignation en tant que manifestation de la désobéissance civique se décline dans notre corpus selon toute une gamme de moyens linguistiques. Si l'on examine les exemples ci-dessous, on voit que ce sont autant d'expressions qu'il faut considérer comme relevant de la subjectivité « affective » multiforme dans la mesure où chacune d'elles - et chacune avec des moyens différents comme dans les exemples (3), (4) et (5) - indique que le sujet d'énonciation se trouve émotionnellement impliqué dans son énoncé.

3. Je suis indigné par le peuple sourd, muet, soumis. Je suis indigné par la condition des femmes dans le monde et en France en particulier.

4. Marre des gros capitalistes de merde !!!

5. Trop de gens se gavent sans que le peuple s'en aperçoive.

Lors de l'analyse des sentiments et des émotions, il faut tout d'abord distinguer les moyens directs et les moyens indirects qui servent à les exprimer. Les chercheurs proposent des typologies des modes de sémiotisation de l'émotion définis en tant que "manières selon lesquelles l'émotion peut être rendue manifeste au moyen de signes » (Micheli, 2013 : 18). Selon Micheli, il existe ainsi trois modes de sémiotisation de l'émotion selon qu'elle est dite, montrée ou étayée 5 . Plantin parle de l'opposition entre les émotions déclarées et inférées. D'autres utiliseront les termes d'émotion dénotée vs connotée. Abstraction faite de ces divergences terminologiques, il reste évident que lorsqu'un individu se trouve confronté au problème de la verbalisation de son état émotif, il peut choisir entre la formulation «directe» où il thématise les émotions éprouvées de même que son implication au moyen de termes affectifs (noms, verbes, adjectifs, adverbes) ${ }^{6}$ (Plantin, 1999) et le discours « subjectif » où il marque sa présence de manière plus ou moins implicite.

Notons aussi que le premier niveau de l'analyse relatif aux dénominations explicites des émotions donne lieu à une distinction supplémentaire. L'énonciation pathémique peut viser à produire un effet de pathémisation de deux manières : d'un côté par la manifestation de l'état émotionnel chez le locuteur, et de l'autre par la description de l'état dans lequel devrait se trouver autrui (ne

${ }^{5} \mathrm{Cf}$ : : « Modes de sémiotisation et fonctions argumentatives des émotions », Semen, 35, Avril 2013, I. Hekmat, R. Micheli, A. Rabatel (dir.)

${ }^{6}$ P.ex. : cruauté, choquant, poignant, insupportable, révoltant, etc. 
craignez rien !, soyez compassif !). Or, la deuxième des variantes mentionnées est surreprésentée dans le corpus par l'injonction « indignez-vous! ».

De manière générale, le matériel textuel réuni n'atteste pas une grande variété de moyens lexicaux véhiculant de l'affectif. Sur le plan du discours transparent et direct, l'effet pathémique est donc obtenu au moyen du recours aux lexèmes emblématiques d'indignation qui représente avec ses dérivés de très nombreuses occurrences.

6. Nous sommes indignés contre un système qui n'est pas durable.

7. Je m'indigne contre la dictature des marchés financiers.

8. Indignée par la médecine à 2 vitesses.

Comme on le voit, si l'indignation peut se décliner selon toute une gamme d'émotions dysphoriques - qui vont de la colère à l'irritation et la frustration -, dans le matériel verbal analysé le répertoire du lexique de l'émotion représentant ce spectre de sentiments est bien limité. Il n'y a guère non plus de somatisation de l'émotion (à quelques exceptions près, puisque nous avons pu trouver dans notre corpus deux occurrences de l'adjectif « écœuré »), l'indignation en tant qu'émotion n'a pas de couleur, etc. Mais, ce qui est à remarquer surtout, c'est que l'indignation en tant que sentiment, donc une entité gradable, ne s'accompagne quasiment pas de marques d'intensification en fonction de la nature des motifs de ressentiment évoqués. Si l'intensification il y a, elle est inférée.

Dans les exemples (10) et (11) on voit que l'émotion dite reste la même quelle qu'en soit la raison en amont.

9. Je suis indigné de ce marché de Noël à côté de nous. On nous vend.

10. Indignée par ce monde sans foi ni loi, ce monde gouverné par l'argent, le nouveau maître, le nouveau Dieu.

Dans l'énoncé pourtant clairement hyperbolisé de l'exemple (11), l'on se contente de la même marque lexicale que dans le cas de l'énoncé précédent. Selon nous, la raison en est la suivante : le manque d'intensifieurs, d'adverbes de complétude (absolument, extrêmement), etc., peut signifier que le mot « indignation " fonctionne ici comme un mot d'ordre, un signe de ralliement d'une communauté et de ce fait ne subit qu'exceptionnellement des modifications.

11. Indignons-nous ! Réveillons-nous !

12. Indignez-vous ! Engagez-vous !

La juxtaposition, très fréquente, des injonctions présentées dans les exemples (12) et (13) montre que l'indignation traduit dans un premier temps une posture, un ethos de l'actant en termes d'engagement acharné dans une situation de ressentiment. 


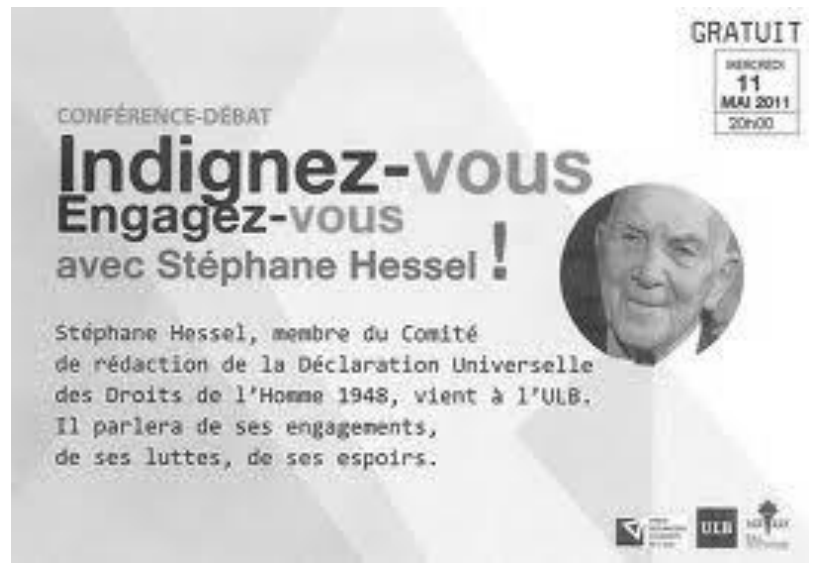

Autrement dit, on la thématise en une conscience-modèle qui se réfère aux normes émotionnelles du groupe. Cela permet aux coénonciateurs de s'inscrire dans un climat d'interaction.

13. « Je vous souhaite à tous, à chacun d'entre vous, d'avoir votre motif d'indignation » (François Hessel).

Le groupe dont il est question ici serait à l'évidence formé par une minorité éclairée et sa posture " engagée » et contestataire représenterait un idéal face à ce qui est présenté comme les abus et la tyrannie de la classe politique et financière corrompue. Voilà pourquoi les manifestations de désaveu s'accompagnent si souvent de la prise en compte des choses de la cité, d'où la récurrence d'isotopies de la communauté et du citoyen.

14. [...] s'autoriser à dire ensemble un « Non » citoyen sur la place publique

15. Et longue vie aux mobilisations citoyennes !

L'acte même de contribuer au blog est donc l'acte de s'engager (ce qui implique du coup un travail émotionnel spécifique), mais aussi d'établir une certaine image de soi, ce qui correspond à une des dimensions intégrantes du discours quel qu'en soit le genre (Amossy, 2010:7). On peut aussi souligner que les utilisateurs essaient souvent d'accroître leur visibilité en insérant des éléments personnels ne serait-ce que sous forme de signature. À côté de l'occurrence la plus fréquente - « citoyen(ne)» s'accompagnant éventuellement d'un qualificatif : p.ex. lambda, ordinaire, etc., on trouve donc assez souvent les lexèmes du type « écœuré », « révolté » ou "scandalisé ». Cela dit, l'affichage trop marqué de l'individualité ne semble pas ici de mise. À la différence des blogs personnels, on ne pourrait parler ici d'écran narcissique, l'accent étant mis sur les topiques de la collectivité dont on se réclame. 
D'un autre côté, nous ne pouvons pas aller jusqu'à considérer que l'émotion est complètement évacuée ; qu'il s'agit là d'un terme d'émotion sans émotion, cognitivement plein, mais « vidé de son contenu affectif» (Plantin, 2012 : 9). Au contraire, l'indignation étant de toute façon vécue comme une émotion ; la visée émotive est bien là, mais nous avons affaire à une sorte de moule pathémique qu'il ne s'agit plus que de remplir d'énoncés exprimant des relations de causalité suivant le choix de la dynamique adopté.

16. Les raisons de nous indigner, nous peuple français en avons plus qu'il n'en faut !

En témoignent par ailleurs les questionnaires préformatés qui invitent les lecteurs/contributeurs des blogs à exprimer leur raisons de l'indignation.

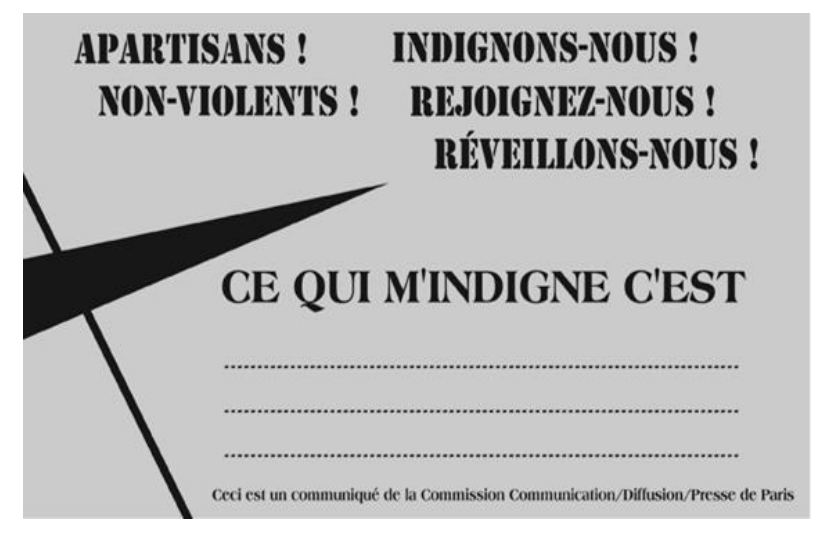

Le cadre de possibles visées pathémiques est bien posé et conditionne le discours du sujet d'énonciation qui peut choisir de les renforcer ou de les atténuer par d'autres moyens, généralement inférés, étoffant ainsi les occurrences du mot « indignation » (cf. infra).

\section{5. ÉMOTION CONNOTÉE / INFÉRÉE}

Comme nous l'avons remarqué plus haut, le corpus analysé est parsemé d'énoncés (ou d'images) susceptibles de construire discursivement un univers pathémique en jouant sur ce qui est sous-jacent à l'expression linguistique. Les émotions y sont inscrites surtout dans des structures évaluatives. A chaque fois que l'on tente d'analyser la manière dont un locuteur représente les émotions, on constate donc le caractère essentiel d'une analyse de type "interprétatif» de l'espace discursif (Maingueneau, 2002). Le deuxième mode de sémiotisation, basé sur les émotions dites montrées ou inférées, nécessite donc une inter- 
prétation à effet de pathémisation (Micheli, 2013 : 31) c'est-à-dire une lecture relative à la fois à des indices amont (situation) et à des indices aval (manifestations de l'émotion) (Plantin, 2012).

Nous devons faire ici une remarque : si l'usage militant des blogs analysés fait des émotions des outils de pathémisation et de mobilisation efficaces, il ne se démarque pas sur ce plan de modes de communication antérieurs. En revanche, la spécificité du support composite réside dans le fait qu'on ne pourrait restreindre le problème aux seules données linguistiques. C'est pourquoi, il est nécessaire de prendre en compte des phénomènes qui vont audelà du code linguistique proprement dit. Ainsi, l'émotion peut être inscrite dans :

- la typographie qui marque l'insistance sur le sens et/ou l'intensité du procès (multiplication des points d'exclamation, lettres grasses ou majuscules) en compensant ce que le textuel perd en termes de prosodie.

17. Il faut changer la donne !!!

18. \#\# Ayrault, Vinci et consorts - le message est clair - Dégagez des terres !

19. ARRETER DE NOURRIR L'HYDRE qui tient la chimie, le nucléaire, les banques, les technologies et institutions

- et le matériel iconique parsemé de binettes (appelées «smiley» ou émoticônes). Ces dernières faisant partie de la sémiologie corporelle sont bien capables de communiquer l'émotion en tant que petits condensés de marques somatiques (en l'occurrence déplaisantes).

Dans ce qui est la couche proprement linguistique, il faut envisager différents indices et procédés relevant du lexical, du champs sémantique, de la morphosyntaxe, des modalités, etc., par lesquels à travers les fonctions expressive et impressive du langage, l'énonciateur manifeste ses sentiments pour agir sur le récepteur. Bien sûr, des procédés variés peuvent se trouver simultanément à l'œuvre dans un même texte. Très souvent on a recours ici à l'emphase, au pathos (persuasion par l'appel à l'émotion) et au procédé d'hyperbolisation afin d'exagérer le sens d'une réalité au moyen d'adjectifs dépréciatifs.

L'émotion inférée peut se traduire notamment par le recours à deux modalités d'énonciation que sont l'exclamation et l'injonction :

20. Cessons de les laisser faire !!!

En témoignent de nombreuses structures phrastiques (la plupart des cas à la $1^{\mathrm{e}}$ personne du pluriel à caractère englobant) repérées. La modalité injonctive dans laquelle s'imbriquent d'ailleurs différentes dimensions - conative, déontique, etc. - donne la primauté à la volonté de mobiliser les récepteurs. Sa tâche est donc pragmatique dans le sens qu'elle accorde la primauté à l'action. 
Les blogs analysés attestent aussi l'emploi très fréquent de l'émotion inférée par le truchement de phrases nominales (parfois réduites à un seul mot: « Non! », " basta! »), de marqueurs dérivationnels (dérivation impropre + satellites), de structures syntagmatiques et phrastiques spécifiques (reduplications, tours emphatiques, etc.) :

21. Partage des richesses !!!

22. Pas de place pour l'oligarchie!!!!

23. La finance c'est assez !

24. Marre de se moquer du monde!

et beaucoup plus rarement à travers l'interprétation de tropes métaphoriques (Meurtre de l'espoir, Les requins des pauvres).

Nous pouvons également repérer un grand nombre d'énoncés proches des slogans à caractère politique déclinés sur un mode traditionnel du type : «non (à quelque chose) », « à bas (quelqu'un) », etc.

25. Non à l'armement manipulateur de conflit.

La modalité exclamative non seulement indique l'intention de communication et manifeste la subjectivité du locuteur, mais elle est également un appel à la réaction de l'interlocuteur et de ce fait possède une dimension intersubjective. Très nombreux, les exclamatifs sont des opérateurs permettant de distribuer des processus cognitifs entre les membres du groupe. Qui plus est, les exemples ci-dessus expriment la frustration et le désir intense de changement, ce qui semble correspondre parfaitement au cadre de la topique des passions aristotélicienne et plus précisément de celle du sentiment d'injustice, fondement de l'indignation.

Le domaine politique a cela de particulier qu'il représente un espace discursif à la fois de mise en relief et de dissimulation. Il en résulte que la nature expressive des énoncés souvent ne se dit pas ouvertement (Damblon, 2001:25). Dans un échange interactionnel, l'émotion peut aussi être simulée, auquel cas, les messages tendent à brouiller la perception du destinataire et l'émotion n'est pas vraie mais seulement vraisemblable. Que l'engagement affectif de l'énonciateur soit réel ou qu'il fasse partie d'une « mise en spectacle discursive » (Rabatel, $2013: 73$ ), il se traduit souvent par le recours à des termes impliquant un jugement de valeur que Kerbrat-Orecchioni (1999: 79) appelle subjectivèmes à la fois « affectifs » et "évaluatifs ». Il s'agit, d'un côté, des évaluatifs qui dénotent clairement des jugements, et de l'autre des connotations d'ordre axiologique attachées à certains lexèmes et dotées d'un potentiel de l'affectivité.

\footnotetext{
${ }^{7}$ Pour de plus amples développements voir P. Breton, La parole manipulée, 1997 (réédition 2004), Paris : La Découverte.
} 
À travers un lexique bien choisi, le locuteur peut en effet augmenter la charge sémantique et pragmatique et mettre simultanément en jeu les processus cognitif et affectif.

Dans la deuxième catégorie mentionnée, nous pouvons relever un grand nombre de verbes (ou leurs dérivés) intrinsèquement subjectifs qui véhiculent une évaluation axiologique dévalorisante soit du procès, soit du comportement comme dans les exemples qui suivent :

26. La Tf1 qui intoxique le peuple et qui fait croire par la puissance médiatique que le monde est rose.

27. [...] certains élus qui pratiquent le copinage et le règlement de comptes sournois.

28. Le discours martelé en permanence par $80 \%$ des médias.

Comme on voit, pour dévaloriser, caractériser hyperboliquement ou intensifier la charge dénigrante, le locuteur a recours à divers procédés lexicosémantiques. Les mêmes procédés peuvent être mis en œuvre par des évaluatifs axiologiques adjectivaux qui énoncent non seulement une propriété de l'objet, mais aussi une réaction émotionnelle du sujet parlant :

29. Changeons les règles d'un jeu pipé !

30. Indigné des politiques véreux, des gens de pouvoir qui mentent au peuple.

On y voit aussi employer les adjectifs « soi-disant» ou «prétendu » qui servent à réfuter la narration adverse.

31. Que font les soi-disant Associations de consommateurs ? (interrogation oratoire)

32. [...] la monnaie, la dette et la prétendue « crise » :

Enfin, les noms et les adjectifs dont la charge sémantique est

- soit clairement péjorative :

33. Le message est toujours le même : ce n'est pas une crise mais un racket !

34. Même les syndicats ont baissé les bras (complices ?)

35. Certains pointent du doigt la collusion des médias principaux avec les pouvoirs en place.

- soit connotée négativement chez les représentants d'une formation discursive donnée : « intérêts », « finance », etc.

De ce point de vue, et cela sans se lancer dans un dépouillement quantitatif du corpus, on peut par ailleurs faire ressortir sans difficulté un lexique qui discrimine, tel un code génétique, les marques idéologiques et partant l'identité politique de la formation discursive en question.

36. [...] nos dirigeants, davantage soucieux des intérêts des banques et autres multinationales que du bien-être de leurs populations. 
37. La droite, ils ont les idées de leurs portefeuilles et de leurs privilèges.

38. La parole des possédants, des détenteurs de l'info.

On voit que le choix des mots, à supposer qu'il soit conscient, participe d'un traitement actif de l'émotion, adjuvant idéologique important, mais cette fois-ci pour donner des signes de reconnaissance et délimiter des territoires linguistiques (Mayaffre, 2003).

\section{CONTRE QUOI ET QUI S'INDIGNER ?}

Certes, l'apparition des évaluatifs axiologiques ne garantit pas qu'ils produisent un effet pathémique, mais une énonciation de la description pathémique qui propose la narration de scènes hyperbolisées est un moyen fréquent de produire un tel effet. C'est d'ailleurs un des éléments canoniques de tout discours politique, surtout populiste: une narration dramatisante de la réalité. Cependant, pour que l'effet recherché puisse apparaître chez le destinataire, il faut que l'on ait recours aux mêmes repères moraux, ces points d'appui que Robrieux appelle «lieux éthiques» (1993: 155). Les émotions présupposent en effet une évaluation basée sur un système polyphonique de valeurs, de croyances - partagées, intériorisées et mobilisées par l'auditoire (imaginaire socio-discursif, réseau inférentiel que peuvent produire les partenaires de l'acte de communication) - et de situations normées ${ }^{8}$. Si nous insistons tant sur l'aspect axiologique, c'est que l'indignation est considérée comme faisant partie des "sentiments moraux » fondés sur des raisons solides (Boudon, 1994 : 47, cité par Amossy, 2006). Or, c'est le caractère objectif et endoxal de celles-ci qui rend tout sentiment d'injustice partageable. Grâce à une définition plus ou moins stable de types d'émotions présentes au sein d'une société et de situations-types qui les déclenchent, on peut décrire le fonctionnement de l'émotion inférée.

On peut d'ailleurs en déduire également d'autres constats. Le processus de rationalisation des émotions, surtout de nature collective, contribue à leur forte dépersonnalisation. On peut par ailleurs remarquer que les émotions morales, support déclencheur à un comportement, apparaissent là où le discours est structuré autour de valeurs polarisées. N'oublions pas que la topique aristotélicienne de l'antipathie est toujours orientée contre quelqu'un et que l'indignation en tant qu'attitude réactive s'accompagne d'un acte de dénonciation et la désignation de coupables, tout en mobilisant un acte de victimisation (Charaudeau, $2000: 151$ ).

\footnotetext{
${ }^{8}$ Nous pouvons nous référer ici à la perspective de la théorie des cadres (Benford et Snow, 2000) selon laquelle l'action collective est le résultat du processus d'ajustement des cadres cognitifs.
} 
Dans le cas concret des Indignés, le schéma d'interprétation qui construit sur une vision partisane et polémique contribue à l'apparition de certains thèmes : ceux de l'oligarchie financière, des élites corrompues, etc.

39. Employer les termes de corruption et corrompus, ça porte dans les consciences !

40. D'entamer une prise de conscience sur la nature oligarchique des systèmes politiques.

\section{OUTIL DE COHÉSION ET DE MOBILISATION}

Nous avons vu que selon Mauss et Durkheim, les émotions sont un caractère social en tant que facteur de cohésion. De manière générale, on peut dire après Damasio (2006), qu'elles déterminent, à tous les niveaux de l'action collective, la prise de décision de l'acteur collectif.

Or, l'indignation en tant que catégorie liée à un jugement partageable au regard des normes, comme d'ailleurs toute émotion dysphorique, poussée à un certain degré peut déboucher sur une action collective, sans être la seule à intervenir. Elle apparaît donc comme un sentiment moteur de la protestation publique et un instrument de remobilisation de la société. Individuelle, elle trouve sa consistance dans la collectivité. (Cf. : la distinction entre les émotions partagées/réciproques de Jasper). Elle peut être considérée comme un instrument, non pas une « passion passive », mais une activité (Plantin, 2012 : 629-642) qui sert à formater la situation d'interaction. Ce procédé peut s'avérer encore plus efficace si, comme c'est le cas des blogs des Indignés, le discours est un discours sur le mode bipolaire entre «nous» et «eux»- souligné par de nombreuses images percutantes - c'est-à-dire avec une énonciation collective impliquant les lecteurs.

41. Ils font passer les intérêts de leurs amis financiers et le leur, avant tout.

42. La dette c'est Eux !!! Eux, c'est les plans de rigueur qui touchent les plus humbles

Aminzade et McAdam (2001) remarquent que l'opposition nous/eux renforce la cohésion et s'accompagne d'une grande charge émotive. Ce «nous » a un caractère éminemment identitaire car il a pour but de célébrer le groupe (énonciation totalisante), d'imposer l'identité plurielle du groupe, qui suggère la pluralité et l'identique, et en fin de compte non pas tant de se constituer en sub-société, mais de souligner que les Indignés représentent les $99 \%$ de la population.

43. Puisque pendant qu'on sert les ceintures d'un coté, les richesses ont augmentés de manière spectaculaire chez les $1 \%$ les plus riches. 


\section{REMARQUES FINALES}

Comme nous l'avons remarqué plus haut, le discours, de par sa nature, éminemment subjectif, peut susciter les émotions. Il n'en reste pas moins vrai que celles-ci représentent un champ d'expérience aux contours imprécis, difficile à conceptualiser. Cela pose devant nous la question non seulement de leur fiabilité dans le contexte du délibératif, mais aussi de leur partage.

À la lumière des exemples présentés, l'indignation des blogs des Indignés apparait comme une sorte de moule pathémique, une catégorie à priori dans laquelle le sujet s'inscrit selon ses affinités en déclinant ses motifs de ressentiment. Dès que ces derniers coulent dans ce moule, il accepte de se positionner en tant que deuxième pôle du jeu spéculaire et de s'assujettir à une certaine entreprise de conviction. Autrement dit, l'émotion est ici donnée en spectacle et discursivement "institutionnalisée ». Le but du procédé consiste non seulement à véhiculer un message, mais aussi à construire un espace identitaire (cf.: Mayaffre, 2003) à travers un contrat de consensus tacite qui facilite l'interaction et implique l'idée d'intentionnalité. Les interactants peuvent ainsi se ressentir comme partie prenante d'une communauté de croyances et de valeurs. Ils ont un rôle à jouer, induits à entrer dans une circulation empathique de l'indignation et à partager l'émotion commune sous peine de démériter. Autrement dit, s'indigner veut dire amorcer une démarche posturale basée sur une certaine doxa quitte à dépathémiser (à force de répéter le lexème « s'indigner ») et à opérer un glissement de la sphère du ressenti et du subjectif vers celle de l'ethos citoyen. On voit par ailleurs que si les blogs peuvent servir d'excellents outils de mobilisation et de sensibilisation, ils sont aussi à même de favoriser des comportements mimétiques. 


\section{BIBLIOGRAPHIE}

Aminzade, R., Mcadam, D. (2001): "Émotions and contentious politics". In : R. Aminzade et al., Silence and Voice in the Study of Contentious Politics. New York: Cambridge University Press, 14-50.

Amossy, R. (dir.) (1999): Images de soi dans le discours. La construction de l'ethos. Paris: Delachaux et Niestlé.

Amossy, R. (2006): L'argumentation dans le discours. Paris : Armand Colin.

AUBERT, A. (2009): " Le paradoxe du journalisme participatif». ENS Cachan. Terrains \& travaux, 15.

CAPT V. et al. (2011): «La figure de l'étranger dans les discours d'un parti politique suisse nationaliste: modes de désignation, traits stéréotypiques et émotions visées ». Revue Interdisciplinaire "Textes \& contextes", 5. [En ligne] http://revuesshs.u-bourgogne.fr/textes\& contextes/document.php?id=1242 ISSN 1961-991X

Cefai D., Pasquier, D., Le Sens public. [En ligne] http://www.u-picardie.fr/labo/curapp/Publi cations/PUFIntro_sens_du_public.pdf

CIGADA, S. (2008): Les Émotions dans le discours de la construction européenne. Milan : Universita Cattolica del Sacro Cuore.

Charaudeau, P. (2000): «Une problématisation discursive de l'émotion, à propos des effets de pathémisation à la télévision ». In : C. Plantin et al. (dir.), Les Émotions dans les interactions. Lyon : ARCI - PUL.

Charaudeau, P. (2007): «De l'argumentation entre les visées d'influence de la situation de communication ». In : C. Boix (dir.), Argumentation, manipulation, persuasion. Paris : L'Harmattan, 13-35.

DAmasio, A. (2006): L'erreur de Descartes. La raison des émotions. Paris : Odile Jacob.

DANBlon, E. (2001): «La rationalité du discours épidictique ». In : M. Dominicy et F. Madeleine (dir.), La mise en scène des valeurs. La rhétorique de l'éloge et du blâme. Lausanne: Delachaux et Niestlé.

DUPERRE, M. (2008): « La rationalité des émotions dans les processus de mobilisation collective ». Service social, 54, 67-81. [En ligne] URI : http://id.erudit.org/iderudit/ 018344ar

KerbRat-Orecchioni, C. (1994): «Rhétorique et pragmatique : les figures revisitées ». Langue française, 101, 57-71.

KeRBRAT-OreCCHIONI, C. (2000): «Quelle place pour les émotions dans la linguistique du XX siècle ? Remarques et aperçus ». In : Ch. Plantin et al. (dir.), Les émotions dans les interactions. Lyon : PUL, 33-74.

MAYAFFre, D. (2003): « Dire son identité politique ». Cahier de la Méditerranée, 66. [En ligne] http://cdlm.revues.org/document119.html

Micheli, R. (2007): "Stratégie de crédibilisation de soi dans le discours parlementaire ». A contrari, 5, 67-84.

MonNoyeR-SMith, L. (2011): « La participation en ligne, révélateur d'une évolution des pratiques politiques? ». Participations, 1, 156-185.

Morin, D. (2003): Émile Durkheim et Marcel Mauss. Étude d'épistémologie historique sur l'émergence de la tradition de recherche des sciences contemporaines, O. Clain (dir, de thèse), Université Laval. [En ligne] http://theses.ulaval.ca/archimede/fichiers/21210/ 21210.html

PENE, S (2007): « Bloguer la politique ». Communication et langages, 151, 73-89.

Plantin, CH. et al. (dir.) (2000): Les émotions dans les interactions. Lyon : ARCI - PUL, 329.

Plantin, CH. (2011): Les bonnes raisons des émotions, Principes et méthode pour l'étude du discours émotionné. Berne : Peter Lang. 
Plantin, CH. (2012): «Les séquences discursives émotionnées : Définition et application à des données tirées de la base CLAPI ». SHS Web of Conferences, Vol. 1, 629-642. [En ligne] http://www.umr8547.ens.fr/IMG/file/ANR\%20Emphiline/Christian\%20Plantin\%20Article.pdf

RoBrieUX, J.-J. (1993): Eléments de rhétorique et d'argumentation. Paris : Dunod.

Van Disk, T. (2006): «Politique, Idéologie et Discours ». Semen, 21, [En ligne] http://semen. revues.org/document1970.html

WierZBicKA, A., JAMroziK, E. (1988): «L'amour, la colère, la joie, l'ennui. La sémantique des émotions dans une perspective transculturelle ». Langages, 89, 97-107. 